\title{
Symptomatic empty sella syndrome: an unusual manifestation of Erdheim-Chester disease
}

\author{
Wann Jia Loh', Kesavan Sittampalam ${ }^{2}$, Suan Cheng Tan $^{3}$ and Manju Chandran ${ }^{1}$ \\ ${ }^{1}$ Osteoporosis and Bone Metabolism Unit, Department of Endocrinology, Departments of ${ }^{2}$ Pathology and \\ ${ }^{3}$ Radiology, Singapore General Hospital, Academia, 20, College Road, Singapore 169856, Singapore
}

Correspondence

should be addressed

to M Chandran

Email

manju.chandran@sgh.com.sg

\section{Summary}

Erdheim-Chester disease (ECD) is a potentially fatal condition characterized by infiltration of multiple organs by nonLangerhans histiocytes. Although endocrine dysfunction has been reported in association with ECD, to date, there have been no previous reports of empty sella syndrome (ESS) associated with it. We report the case of a patient with ECD who had symptomatic ESS. A 55-year-old man of Chinese ethnicity initially presented with symptoms of heart failure, fatigue and knee joint pain. Physical examination revealed xanthelasma, gynaecomastia, lung crepitations, hepatomegaly and diminished testicular volumes. He had laboratory evidence of hypogonadotrophic hypogonadism, secondary hypoadrenalism and GH deficiency. Imaging studies showed diffuse osteosclerosis of the long bones on X-ray, a mass in the right atrium and thickening of the pleura and of the thoracic aorta on fusion positron emission tomography-computed tomography. Magnetic resonance imaging (MRI) of the brain showed an empty sella. The diagnosis of ECD was confirmed by bone biopsy.

\section{Learning points:}

- ECD is a multisystemic disease that can affect the pituitary and other organs. The diagnosis of ECD is based on clinical and radiological features and histology, showing lipid-laden $\mathrm{CD} 68^{+} \mathrm{CD} 1 \mathrm{a}^{-} \mathrm{S} 100^{-}$histiocytes surrounded by fibrosis.

- The finding of xanthelasmas especially in the presence of normal lipid levels in the presence of a multisystem infiltrative disorder should raise the suspicion of ECD.

- Systemic perturbation of autoimmunity may play a role in the pathogenesis of ECD and is an area that merits further research.

\section{Background}

Erdheim-Chester disease (ECD) remains a challenging case to diagnose and treat. ECD should be considered in patients with hypogonadotropic hypogonadism and hypopituitarism on a background of multisystem infiltrative involvement. Thyroid infiltration is also extremely rare in ECD with only one case of thyroiditis associated with it reported thus far. The association that was found with Hashimoto's thyroiditis as in this case highlights the importance of further research that should be undertaken with regard to autoimmunity as a possible underlying mechanism in the development of this fascinating disease.

\section{Case presentation}

A 55-year-old Chinese man presented to the hospital with fatigue, knee joint pains and breathlessness on exertion that had been worsening over the last few weeks. On examination, he was found to be in fluid overload with bilateral lung crepitations, a raised jugular venous pressure and pitting oedema up to midshin. It was noted that he 


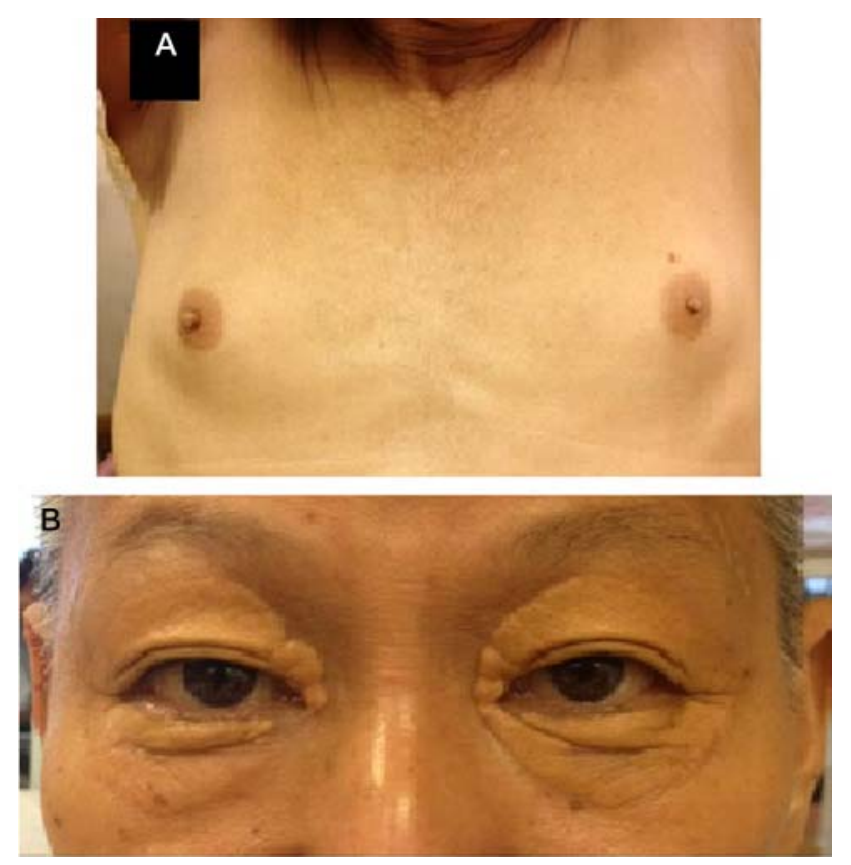

Figure 1

Physical examination of the patient revealing (A), bilateral gynaecomastia and (B) xanthelasma.

also had non-tender hepatomegaly, gynecomastia and bilateral xanthelasma (Fig. 1). Both testes showed diminished testicular volumes. Initial routine laboratory investigations including haemoglobin and haematocrit were normal as was his renal function. There were no electrolyte abnormalities nor were there any osmolar disturbances in the serum or urine. Total cholesterol, $\mathrm{HDL}$, triglyceride and LDL levels were $3.29 \mathrm{mmol} / \mathrm{l}$, $0.47 \mathrm{mmol} / \mathrm{l}, 1.47 \mathrm{mmol} / \mathrm{l}$ and $2.15 \mathrm{mmol} / 1$ respectively on a fasting lipid panel. A two-dimensional echocardiogram showed that the ejection fraction was $70 \%$ and the heart valves were unremarkable. A small $2.5 \times 1.3 \mathrm{~cm}$ mass was noted on the posterior wall of the right atrium extending superiorly. There was no pericardial effusion. Holter monitoring was done for $24 \mathrm{~h}$, and it revealed tachy-brady syndrome, atrial fibrillation and long pauses of up to $4.8 \mathrm{~s}$. This cardiac dysrhythmia likely explained his congestive cardiac failure.

He recalled that the thickening around his eyelids had started appearing about 10 years ago and that 7 years ago he had a significant history of polydipsia and polyuria for 1 year. He drank 4-5 1/day and passed urine more than ten times per day, awakening four to five times every night to pass urine. He did not visit a doctor then. The symptoms spontaneously resolved without treatment. He stated that he had noticed breast enlargement a few years ago but had not sought medical attention for it nor for the reduced early morning erections or decreased libido he had been experiencing for years. He has two children.

\section{Investigation}

Computed tomography (CT) scan of the body showed soft tissue thickening around the pericardium, thoracic aorta (Fig. 2), coronary arteries and left renal artery. There was also pleural thickening, pulmonary fibrosis and retroperitoneal lymphadenopathy. A skeletal X-ray survey showed widespread osteosclerosis of the long bones (Fig. 2).

Endocrine evaluation showed multiple anterior pituitary hormone deficiencies with hypogonadotropic hypogonadism, secondary adrenal insufficiency and
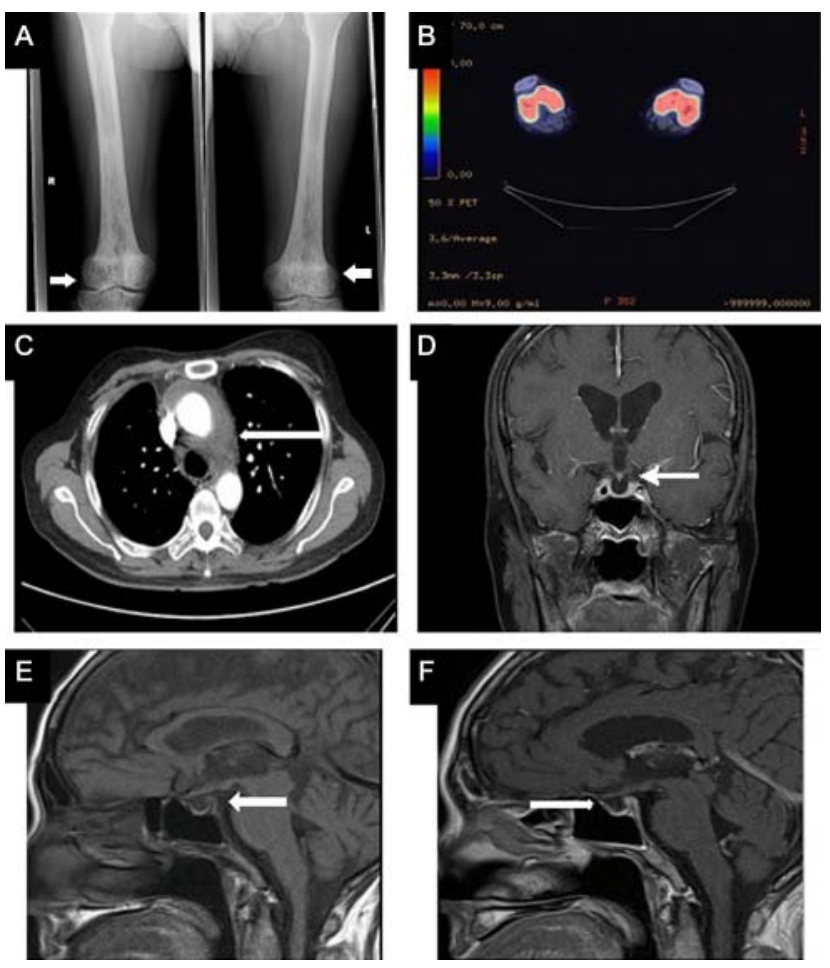

Figure 2

Radiological imaging. (A) Thickened and sclerotic trabeculae with intervening lucencies of both femurs (arrow). (B) Fusion image of PETcomputed tomography (CT) scan at the femoral condyles with intense F-18 fluorodeoxyglucose (FDG) uptake in keeping with increased metabolic activity. (C) CT thorax (axial views) with the abnormal soft tissue thickening surrounding the ascending aorta (arrow). (D) T1-weighted contrasted coronal MRI pituitary image with the central pituitary stalk (arrow). (E) Sagittal weighted T1 image of the MRI pituitary before contrast medium administration. There is loss of the normal T1 signal hyperintensity of the posterior pituitary lobe (thick arrow). (F) Sagittal weighted T1 MRI pituitary image after contrast medium administration. The pituitary gland is thinned out and flattened at the floor of the pituitary fossa (thin arrow). 
Table 1 Results of endocrinological investigations.

\section{Variable}

Follicle-stimulating hormone $(\mathrm{mlU} / \mathrm{ml})$

Luteinizing hormone $(\mathrm{mlU} / \mathrm{ml})$

Testosterone levels

Total testosterone $(\mathrm{nmol} / \mathrm{l})$

Free calculated testosterone $(\mathrm{nmol} / \mathrm{l})$

Free calculated testosterone (\%)

Sex hormone-binding globulin ( $\mathrm{nmol} / \mathrm{l})$

Oestradiol (pmol/l)

Cortisol after $1 \mu \mathrm{g}$ tetracosactide $(\mathrm{nmol} / \mathrm{l})$; reference $>500 \mathrm{nmol} / \mathrm{l}$

$0800 \mathrm{~h}$ cortisol levels ( $\mathrm{nmol} / \mathrm{l})(0 \mathrm{~min})$

Cortisol levels $15 \mathrm{~min}$ after $1 \mu \mathrm{g}$ corticotrophin $(\mathrm{nmol} / \mathrm{l})$

Cortisol levels $30 \mathrm{~min}$ after $1 \mu \mathrm{g}$ corticotrophin (nmol/l)

Cortisol levels $45 \mathrm{~min}$ after $1 \mu \mathrm{g}$ corticotrophin ( $\mathrm{nmol} / \mathrm{l}$ )

$\mathrm{ACTH}(\mathrm{ng} / \mathrm{l})$ at $0 \mathrm{~min}$

Insulin-like growth factor $(\mu \mathrm{g} / \mathrm{l})$

Growth hormone (GH) (mU/l)

Prolactin $(\mu \mathrm{g} / \mathrm{l})$

Thyroid-stimulating hormone $(\mathrm{mlU} / \mathrm{l})$

Free thyroxine (pmol/l)

Anti-thyroglobulin antibody titre (IU/ml)

\section{Reference range, adults}

$1.2-8.1$
$2.1-10.9$
$7.3-27.4$
$0.180-0.994$
$10.5-61$
$44-156$
$123-626$


$10-60$
$88-262$
$0-28.5$
$5-27.7$
$0.27-4.2$
$11.8-24.6$
$10-115$

Patient's value

0.2

0.1

2.1

0.0319

1.52

53

188

219

305

364

339

54.1

39.5

1

51.1

16.2

11

939 growth hormone $(\mathrm{GH})$ deficiency (Table 1). Interestingly, his thyroid function testing revealed primary hypothyroidism with free thyroxine level of 11 (11.8-24.6) pmol/1 and thyroid-stimulating hormone level of 16.2 (0.27-4.2) $\mathrm{mIU} / \mathrm{l}$. His anti-thyroglobulin antibody titre was significantly raised at 939 (10-115) IU/ml.

A magnetic resonance imaging (MRI) of the brain and pituitary showed features of the empty sella (Fig. 2). The pituitary gland was thinned out and flattened. The pituitary stalk was central. There was loss of T1 signal hyperintensity of the posterior pituitary. The sella turcica was not enlarged. A fusion positron emission tomography (PET)-CT scan showed multisystem involvement with hypermetabolic activity in the long bones, pericardial soft tissue and perivascular soft tissue.

The patient underwent a bone biopsy of the femur, which showed small clusters of CD68 and CD163 immunopositive foamy macrophages. S100 and CD1a immunostaining was negative (Fig. 3). This is diagnostic of ECD (1).

\section{Treatment}

Although the complete mechanism of action still remains to be elucidated, pegylated interferon (IFN) alpha-2a has been shown to improve survival rates in this disease, and this was initiated at a dose of $180 \mu \mathrm{g}$ subcutaneously once a week. Daily oral prednisolone at a dose of $50 \mathrm{mg}$ daily was started. In view of his symptomatic hypogonadism, he was also started on transdermal testosterone gel $1 \%$ daily. He was also started on levothyroxine $50 \mu \mathrm{g}$ daily for his primary hypothyroidism. A cardiac pacemaker was inserted for his dysrhythmia. The patient was tested positive for $B R A F$ mutation; however, the clinical significance to the treatment of ECD still remains investigational.

\section{Outcome and follow-up}

After 1.5 years, his disease remains stable and there has not been any recurrence of pain and breathlessness since therapy was started. He is working full time. The repeat PET-CT scan at 6 months post-therapy showed partial metabolic response at all the skeletal lesions, and stable lesions in the pericardium, pleura and arteries. No new hypermetabolic lesions were noted. He is still on tapering

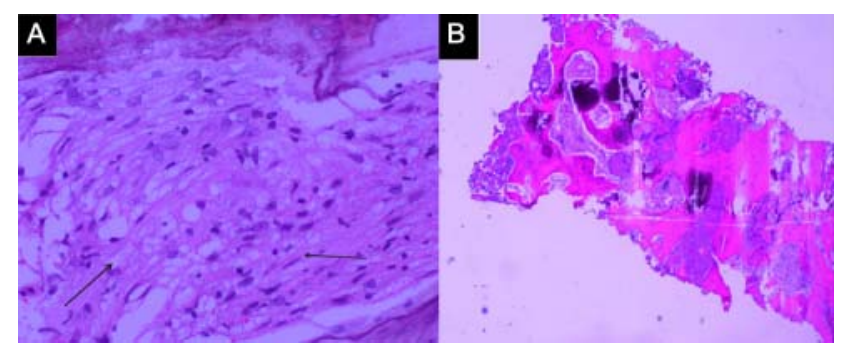

\section{Figure 3}

Histology of bone biopsy specimen. (A) High power view of foamy macrophages in the interlamellar spaces (thin arrow), using $\mathrm{CD}_{163}{ }^{+} \mathrm{CD} 68^{+}$ $\mathrm{CD} 1 \mathrm{a}^{-} \mathrm{S} 100^{-}$immunostain; $200 \times$ magnification. (B) Low-power view of trabeculae of woven and lamellar bone, $100 \times$ magnification. 
dose of glucocorticoids as well as regular pegylated IFN alpha-2a, levothyroxine and i.m. testosterone undecanoate injections. His thyroid function normalized within 3 months of initiation of levothyroxine. As the thyroid pathology appeared to be primary in nature, re-testing the thyroid axis off levothyroxine would not be helpful or necessary. Re-evaluation of the patient's hypothalamopituitary-gonadal axis, GH axis and hypothalamopituitary-adrenal axis may be considered in the future if the patient is weaned off immunomodulator treatment and glucocorticoids.

\section{Discussion}

Although increasingly being identified, ECD still remains a rare entity and its diagnosis remains challenging. The disease can range from asymptomatic skin lesions to lifethreatening multi-organ failure. The diagnosis of ECD is based on clinical and radiological features and histology showing lipid-laden $\mathrm{CD}^{+} 8^{+} \mathrm{CD} \mathrm{a}^{-} \mathrm{S}_{100}{ }^{-}$histiocytes surrounded by fibrosis (1).

Although bone involvement is often the most characteristic sign of ECD, foamy histiocytic infiltration of any organ including the pituitary can occur (1). Hypothalamic involvement causing hypogonadotrophic hypogonadism as well as GH deficiency and hypeprolactinaemia have also been reported (2). Hypogonadotrophic hypogonadism tends to occur early in the disease. He had a flattened and thinned out pituitary gland characteristic of the empty sella syndrome (ESS). This has not been reported previously with ECD even after years of follow-up.

ESS is a condition in which the sella turcica is partially or completely filled with cerebrospinal fluid (CSF), resulting in a displacement of the pituitary gland. It is characterized by distinct radiological and anatomical features (3). The condition may be primary or secondary. Secondary causes include previous pituitary surgery, radiotherapy and medical treatment for tumours of sellar region.

Endocrinological dysfunction is not common in primary ESS with it being reported in about $25-35 \%$ of patients (4). Our patient had hypopituitarism with hypogonadotropic hypogonadism, central hypocortisolism (inappropriately normal ACTH level with low baseline and stimulated cortisol levels) and GH deficiency. Mild hyperprolactinaemia has been associated in 15\% of ESS, possibly due to pituitary stalk stretching (3).

Although thus far not been reported in ECD, ESS has been noted to occur in association with Langerhans histiocytosis (5) and granulomatous disorders such as sarcoidosis (6). It appears that the timing to progression to ESS is variable ranging from 12 months to 20 years after onset of the primary disease (5) (6) (7). The speed of progression may be faster if ischaemic flow to the pituitary causing infarction occurred (7). This does not appear to have been the case in our patient nor did he have any signs of raised intracranial pressure - another postulated mechanism of ESS (3). He did not have MRI findings of a previous cerebrovascular accident nor did he have any history of symptoms or signs suggestive of pituitary apoplexy or infarction. We postulate that in our patient, histiocytic infiltration of the pituitary must have been active in the early years of his ECD. He had loss of normal T1 enhancement of the posterior pituitary on MRI. Although this finding may be non-specific, the history the patient gave suggestive of a transient diabetes insipidus (DI) seven years ago gives it added weightage. This is corroborated by rare reports of patients presenting with DI 6-18 years before the diagnosis (1). The posterior pituitary consists of only the distal axons of the hypothalamic nucleus and so, lesions of the posterior pituitary rarely cause permanent DI since hypothalamic nuclei can still produce and secrete vasopressin directly into circulation. This appears to have been the case in our patient also. Subsequent destruction and flattening of the pituitary with development of pan-hypopituitarism may have resulted in the atrophy of the pituitary gland, which created space for the subarachnoid herniation and the finding of ESS on MRI.

Thyroid infiltration is rare in ECD with only one case of Hashimoto's thyroiditis reported in association with ECD (8). Our patient also has likely autoimmune thyroiditis in view of his raised anti-thyroglobulin antibody levels. Although the issue of whether the pathogenesis of ECD is due to a monoclonal neoplastic process or whether it is due to a polyclonal immune mediated one remains debatable and controversial, our finding sheds interesting light on the possible role of a common systemic perturbation in autoimmunity in the pathogenesis of both ECD and Hashimoto's thyroiditis. Although earlier case reports seem to suggest monoclonal proliferation as a potential mechanism in ECD, more recent work has shown alterations in cytokine and chemokine networks in ECD such as tumour necrosis factor alpha (TNF $\alpha$ ), interleukin 6 (IL6), CXCL8/IL8 and IFN $\alpha$, which were postulated to activate histiocytes in the disorder (9). T-helper 1 cells predominate in Hashimoto's thyroiditis (10) and also in ECD (9). However, expression of cytokines involved in these two diseases is different. 
ECD is associated with high systemic levels of IL6, TNF $\alpha$ and low IL4 (9) whereas Hashimoto's thyroiditis is associated with raised IFN $\gamma$ and IL2 levels (10). The role of unidentified pituitary antibodies in the development of the ESS in our patient also cannot be ruled out.

Skin infiltration in ECD causes xanthelasma and xanthomas, which are useful clues to this disease. This has been reported to be present at the onset in $11 \%$ of patients with the syndrome (2). Recognising that xanthelasma can be a presentation of this disease especially when associated with normal lipid levels may help prevent the diagnostic delay that has been reported to occur in this rare and challenging disorder.

\section{Declaration of interest}

The authors declare that there is no conflict of interest that could be perceived as prejudicing the impartiality of the research reported.

\section{Funding}

This research did not receive any specific grant from any funding agency in the public, commercial or not-for-profit sector.

\section{Patient consent}

Written informed consent has been obtained from the patient for publication of the case report and accompanying images.

\section{Author contribution statement}

Dr W J Loh is the physician taking care of the patient and who wrote the case report. Dr K Sittampalam was involved in analysing the histology slides and critical revision of the paper. Dr S C Tan was involved in bone biopsy, interpretation of the radiological images and critical revision of the paper. Dr M Chandran was involved in the conception, design, final approval and critical revision of the paper.

\section{References}

1 Veyssier-Belot C, Cacoub P, Caparros-Lefebvre D, Wechsler J, Brun B, Remy M, Wallaert B, Petit H, Grimaldi A, Wechsler B et al. 1996 Erdheim-Chester disease. Clinical and radiologic characteristics of 59 cases. Medicine 75 157-169. (doi:10.1097/00005792-199605000-00005)

2 Tritos NA, Weinrib S \& Kaye TB 1998 Endocrine manifestations of Erdheim-Chester disease (a distinct form of histiocytosis). Journal of Internal Medicine 244 529-535. (doi:10.1111/j.1365-2796. 1998.00389.x)

3 Agarwal JK, Sahay RK, Bhadad SK, Reddy VS \& Agarwal NK 2001 Empty sella syndrome. Journal of Indian Academy of Clinical Medicine 2 198-202.

4 Gallardo E, Schächter D, Cáceres E, Becker P, Colin E, Martínez C \& Henríquez C 1992 The empty sella results of treatment in 76 successive cases and high frequency of endocrine and neurological disturbances. Clinical Endocrinology 37 529-533. (doi:10.1111/j.1365-2265.1992. tb01484.x)

5 Balaguruswamy S \& Chattington PD 2011 Partial hypopituitarism and Langerhans cell histiocytosis. BMJ Case Reports 2011 bcr0720103203. (doi:10.1136/bcr.07.2010.3203)

6 Chiang R, Marshall MC, Jr, Rosman PM, Hotson G, Mannheimer E \& Wallace EZ 1984 Empty sella turcica in intracranial sarcoidosis. Pituitary insufficiency, primary polydipsia, and changing neuroradiologic findings. Archives of Neurology 41 662-665. (doi:10.1001/archneur. 1984.04210080070017)

7 Lin TJ, Hwang FC, Chiu WT, Lin JW, Tsai SH \& Chang CK 2005 Empty sella hypogonadism and hypopituitarism secondary to moyamoya disease. Journal of Clinical Neuroscience 12 472-474. (doi:10.1016/j.jocn. 2004.05.022)

8 Cruz AA, Alencar VM, Falcão MF, Elias J \& Chahud F 2006 Association between Erdheim-Chester disease, Hashimoto thyroiditis, and familial thrombocytopenia. Ophthalmic Plastic and Reconstructive Surgery 22 60-62. (doi:10.1097/01.iop.0000197021.98742.09)

9 Arnaud L, Gorochov G, Charlotte F, Lvovschi V, Parizot C, Larsen M, Ghillani-Dalbin P, Hervier B, Kahn JE, Deback C et al. 2011 Systemic perturbation of cytokine and chemokine networks in Erdheim-Chester disease: a single-center series of 37 patients. Blood 117 2783-2790. (doi:10.1182/blood-2010-10-313510)

10 Fisfalen ME, Palmer EM, Van Seventer GA, Soltani K, Sawai Y, Kaplan E, Hidaka Y, Ober C \& DeGroot LJ 1997 Thyrotropin-receptor and thyroid peroxidase-specific $\mathrm{T}$ cell clones and their cytokine profile in autoimmune thyroid disease. Journal of Clinical Endocrinology and Metabolism 82 3655-3663.

Received in final form 25 February 2015

Accepted 10 March 2015 